\title{
Secondary Hemophagocytic Syndrome: Differences between the etiologies autoimmunes and hematological malignancies subgroup
}

César Antonio Egües Dubuc ( $\nabla$ tonoeguesdubuc@hotmail.com )

Hospital Universitario de Donostia https://orcid.org/0000-0002-3637-1239

Jaime Calvo-Alen

Hospital Universitario Araba

Lizbeth Patricia Cabrera-Miranda

Hospital Universitario 12 de Octubre Centro de Investigacion Biomedica

Andrea De Diego Sola

Hospital Universitario de Donostia

José Ramon Furundarena Salsamendi

Hospital Universitario de Donostia

Nerea Alcorta Lorenzo

Hospital Universitario de Donostia

Jesús Alejandro Valero Jaimes

Hospital Universitario de Donostia

Luis María López Dominguez

Hospital Universitario de Donostia

Jorge Jesús Cancio Fanlo

Hospital Universitario de Donostia

Olga Maiz Alonso

Hospital Universitario de Donostia

Esther Uriarte Isacelaya

Hospital Universitario de Donostia

Joaquín María Belzunegui Otano

Hospital Universitario de Donostia

\section{Research}

Keywords: Hemophagocytic Syndrome, Hemophagocytic Lymphohistiocytosis, Autoimmune diseases, Hematological Malignancies.

Posted Date: June 29th, 2020 
DOI: https://doi.org/10.21203/rs.3.rs-37968/v1

License: (c) (i) This work is licensed under a Creative Commons Attribution 4.0 International License. Read Full License 


\section{Secondary Hemophagocytic Syndrome. Differences between the etiologies autoimmunes and hematological malignancies subgroup}

César Antonio Egües Dubuc ${ }^{1}$, M.D.; Jaime Calvo-Alen², M.D., PhD; Lizbeth Patricia Cabrera-Miranda ${ }^{3}$, M.D.; Andrea De Diego Sola ${ }^{1}$, M.D.; José Ramon Furundarena Salsamendi", M.D.; Nerea Alcorta Lorenzo ${ }^{1}$, M.D.; Jesús Alejandro Valero Jaimes ${ }^{1}$, M.D.; Luis María López Dominguez¹, M.D.; Jorge Jesús Cancio

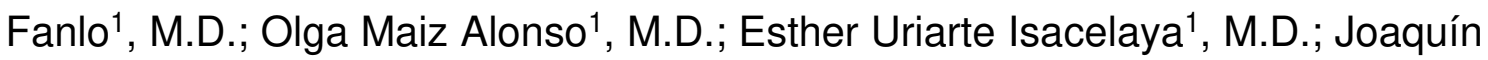
María Belzunegui Otano' ${ }^{1}$, M.D.;

1.-Donostia University Hospital, Rheumatology, Donostia, Spain.

2.-Araba University Hospital, Rheumatology, Vitoria, Spain.

3.-University Hospital October 12, Preventive Medicine and Public Health, Madrid, Spain.

4.-Donostia University Hospital, Hematology, San Sebastian, Spain.

\section{Corresponding autor:}

- César Antonio Egües Dubuc

- Institution: Donostia University Hospital. Begiristain Doktorea Pasealekua, 109, 20014 Donostia, Gipuzkoa, Spain.

- Address: Paseo de Ayete 19, 4 izq. 20009 Donostia, Gipuzkoa, Spain.

- Telephone: $0034-626513576$

- E-mail address: tonoeguesdubuc@hotmail.com 


\section{Abstract:}

Background: Hemophagocytic Syndrome (HPS) is an uncommon disorder with high mortality. HPS is reported to be usually related to some underlying conditions; autoimmune ( $\mathrm{Al}$ ) and hematological malignancies (HM) are two main underlying conditions. Here, we attempted to determine the clinical characteristics/differences of HPS according to underlying conditions; Al vs. HM, based on our experience.

Methods: We studied all adult patients with HPS diagnosed at our institution from 2005 to 2019. Demographic, clinical characteristics, laboratory findings, underlying disorders, hospital stay and prognosis variables were examined and related to the underlying conditions.

Results: 30 patients (median age of 55 years and male/female of 14/16) met the inclusion criteria which we analyzed: HM: 12 (40\%), Al: 10 (33\%), and others: 8 (27\%). HM group, compared with Al group, showed a significantly longer length hospital stay (HM vs Al: 61.5 vs 30.5 (median)), more severe thrombocytopenia, leukocytopenias, and neutropenia, and importantly, higher mortality (with overall mortality and HM-related HPS-mortality being 43.3 and $66.7 \%$, respectively). Contrarily, HM group, compared with Al group, showed less severe liver abnormalities (AST; 106 vs 457 (median): ALT; 109 vs 457 (median)).

Conclusions: The HM subgroup presented a greater mortality and a higher number and severe cytopenias but lower elevation of transaminases.

Keywords: Hemophagocytic Syndrome, Hemophagocytic Lymphohistiocytosis, Autoimmune diseases, Hematological Malignancies. 


\section{List of abbreviations in alphabetical order:}

- A.P.: alkaline phosphatase

- Al: Autoimmune

- ALT: alanine aminotransferase

- ASD: Adult Still's Disease (ASD).

- AST: aspartate aminotransferase

- B.T.: total bilirubin

- BM: Bone marrow

- CMV: Cytomegalovirus

- CSA: Cyclosporine A

- EBV: Epstein Barr

- Fb: fibrinogen

- Fer: ferritin

- GC: Glucocorticoids

- GGT: gamma-glutamyl transferase

- GM: Glioblastoma multiforme

- GSR: Globular sedimentation rate

- Hb: hemoglobin

- HC: Hemophagocytic cells

- HIV: Human Immunodeficiency Virus

- HLH: Hemophagocytic Lymphohistiocytosis

- HM: Hematological Malignancies

- HPS: Hemophagocytic Syndrome

- IL: interleukins

- LDH: lactate dehydrogenase

- INR: International normalized ratio

- JIA: Juvenile Idiopathic Arthritis

- Leu; leukocytes

- MAS: Macrophage Activation Syndrome

- MST: Malignant solid tumors

- Neu, neutrophils

- NK: Natural Killer 
- Pt: platelets

- SG: subgroup

- SLE: Systemic Lupus Erythematosus

- T.P.: total protein

- Tg: triglycerides 


\section{Declarations}

Ethic's approval and consent to participate: The study was approved by the ethics committee of the Donostia University Hospital, and given the retrospective nature of this study; it was not necessary to obtain informed consent, as the ethics committee did not require it or waived it. However, after data collection, patients were anonymized. In addition, the study followed the guidelines of the Organic Law 3/2018, of December 5, on the Protection of Personal Data and Guarantee of Digital Rights, which repealed the Organic Law 15/1999, of December 5, on the Protection of Personal Data in Spain.

Consent for publication: Consent was not obtained because the retrospective nature of the study.

Availability of data and materials: Not applicable.

Competing interests: The authors declare that they have no competing interests.

Funding: Funding research wasn't received.

\section{Authors' contributions:}

CAED: Main author. Major contributor in writing the manuscript.

JCA: Second main author. Contributor in writing and review the manuscript.

PCM: Third main author. Performed the statistical analysis and review the manuscript.

ADDS: Helped to make the introduction and to collected information from medical records.

JRFS: Performed the reading and analysis of the bone marrows and collected information from medical records.

NAL, JAVJ, LMLD, JJCF, OMA, EUI: Collected information from medical records JMB: Review the manuscript. 
Also, all authors read and approved the final manuscript.

Acknowledgements: Not applicable. 


\section{Introduction:}

Hemophagocytic Lymphohistiocytosis (HLH), also called Hemophagocytic Syndrome (HPS), is characterized by a clinical, inflammatory, acute and severe syndrome with high mortality without early treatment. To refer to this entity, in this study, we will use exclusively the acronym "HPS". The first cases of HPS in children and adults were published in 1952 and 1979 respectively. (1,2) The incidence of HPS is unknown, but in one hospital of Sweden the incidence was 0.12/100,000 patient/year for familial HPS and 0.36/100,000 patient/year for HPS associated with malignancy. (3) The HPS is expressed by a proliferation and activation of $\mathrm{T}$ cells and macrophages, producing a hypersecretion of cytokines such as interferon-gamma, tumor necrosis factor-alpha, interleukins (IL) 1, IL-6, IL-10, IL-12, IL-18 and macrophage colony-stimulating factor. $(4,5)$

The HPS has been classified into 2 groups: primary or genetic, and secondary or acquired. Primary, usually called Familial Hemophagocytic Lymphohistiocytosis, is a rare disease that commonly affects infants and children. $(5,6)$ It is inherited in an autosomal recessive manner, and 5 subtypes are known. (7) The secondary form is mainly associated with hematological malignancies (HM) such as lymphomas or leukemias, and autoimmune $(\mathrm{Al})$ diseases such as Systemic Lupus Erythematosus (SLE) and Child or Adult Still's Disease (ASD). The HPS secondary to Al disease is called Macrophage Activation Syndrome (MAS). Less frequently, HPS can be associated with infections, either as etiology of HPS or as a trigger over Al or HM diseases. Others less frequent causes of secondary HPS are transplants, malignant solid tumors (MST) due to the production of cytokines by itself or under chemotherapy. $(4,8)$

The most common manifestations are fever, hepatosplenomegaly, altered liver and coagulation function, hypertriglyceridemia, cytopenias, hyperferritinemia and the histological presence of hemophagocytic cells (HC) in bone marrow (BM), spleen or lymph nodes. (4)

We are currently facing a rare entity with a high mortality rate. The diagnosis is complex and can affect different profiles of patients treated by various medical specialists, so having a basic knowledge of this entity, knowing the various clinical 
manifestations, laboratory findings, diagnostic criteria and differential diagnostic procedures can favor a timely approach. Autoimmune disorders and malignancy are two important underlying causes of HPS; however, clinical characteristics of HPS according to the underlying disorders are not well determined. Although there have been important advances in recent years, but are insufficient. The most relevant advances are the recommendations made by the American Society of Hematology and a scoring system to predict the probability of diagnosing HPS made by the American College of Rheumatology, published in 2019 and 2014 respectively. $(8,9)$ The present study was an effort to determine this issue based on our own experience describing demographic, clinical manifestation, laboratory findings and prognostic characteristics according to underlying conditions (Al vs. HM)

\section{Material and methods:}

We retrospectively analyzed a cohort of 30 adult patients over 17 years old with diagnosis of HPS during their admission in the Donostia University Hospital between December 2005 and October 2019, based on the HLH-2004 criteria from the Histiocyte Society, or presented HC in the BM biopsy, or who had HPS diagnosis in the hospital discharge report. The diagnosis based on the HLH-2004 criteria required to meet at least five of the following criteria: 1.- prolonged fever (a temperature $\geq 38.5{ }^{\circ} \mathrm{C}$ for $\geq 7$ days), 2.- splenomegaly (the costal margin exceeded $3 \mathrm{~cm}$ ), 3.- cytopenia involving in at least two lineages of peripheral blood (neutrophil count $<1.0 \times 109 / \mathrm{L}$, hemoglobin $<90 \mathrm{~g} / \mathrm{L}$ or platelet $<100 \times$ $109 / \mathrm{L}$ ), 4.- hypertriglyceridemia (fasting triglycerides $\geq 3 \mathrm{mmol} / \mathrm{L}$ ) and/or hypofibrinogenemia (fibrinogen $\leq 1.5 \mathrm{~g} / \mathrm{L}$ ), 5.- hemophagocytosis in bone marrow, spleen or lymph nodes, 6 .- low or absent NK cells activity, 7.- serum ferritin $\geq 500$ $\mu \mathrm{g} / \mathrm{L}$ and 8.- soluble CD25 (soluble interleukin-2 receptor) $\geq 2400 \mathrm{IU} / \mathrm{ml}$. (4) However, the tests for soluble CD25 levels and NK cell activity were not available in our institution.

The study was approved by the ethics committee and given the retrospective nature of this study; informed consent was not obtained. However, after data 
collection, patients were anonymized. In addition, the study followed the guidelines of the Organic Law 3/2018, of December 5, on the Protection of Personal Data and Guarantee of Digital Rights, which repealed the Organic Law 15/1999, of December 5, on the Protection of Personal Data in Spain.

The demographics, clinical characteristics (etiology, underlying disorders, diagnostic criteria and hospital stay), relevant laboratory findings (complete blood count, biochemical profile, coagulation, ferritin and others), treatments and prognosis were extracted from the electronic medical record; examined and related to the underlying conditions.

Data analysis was performed using SPSS 21.0 software. Categorical variables are shown as frequencies and percentages ( $n, \%)$. Continuous variables are presented with the mean $(\mathrm{x} \pm \mathrm{s})$ or median [interquartile rank (IQR)] according to the degree of normality of the variable distribution. Non-parametric tests were used for most quantitative variables because the abnormal distribution. The analysis of inter-group differences (qualitative variables) was performed using the Fisher's test. The Kruskal Wallis' test was used in the analysis between continuous quantitative variables and the polynomial categorical variable "etiology". The analysis between continuous quantitative variables and the analysis of the 2 subgroups ( $\mathrm{Al}$ and $\mathrm{HM}$ ) the Mann-Whitney $\mathrm{U}$ test were used.

\section{Results:}

Patient characteristics, etiologies, underlying disorders and treatments

Thirty patients with diagnosis of HPS were included [ 16 females; mean age, 55.5 years ( \pm 18.3 years)]. Patients were distributed in 5 subgroups according to the etiology: AI, HM, MST, Infectious and HPS without defined etiology (Table 1). The debut of the primary etiology coincided with the diagnosis of HPS in $12(57 \%)$ patients, and it was predominant in Al subgroup (60\%). The coincidence of an infectious disease with the diagnosis of HPS was observed in 10 (33.3\%) patients, 2 were considered to be the primary etiology of HPS and 8 were considered to be the trigger of HPS [AI: 5 ( $2 \mathrm{CMV}, 2$ probably viral respiratory 
infections and 1 bacterial infection) and HM: 3 (2 EBV and 1 bacterial infection)]. In 2 patients with acute leukemia the allogeneic transplantation was associated as the trigger of HPS. In one patient with myelodysplastic syndrome the HPS was associated with the development of graft-versus-host disease. Finally, in one patient with glioblastoma multiforme (GM) the chemotherapy (temozolomide) was considered the trigger event.

Five patients were included in the HPS without defined etiology subgroup. Three died from HPS during hospital admission. The other 2 cases continue to be followed by the Hematology Service without finding an etiological cause of the HPS (1523 and 1573 days of follow-up after discharge).

Only one patient presented a reactivation of HPS (36 days after discharge). The diagnosis of first hospital admission was HPS secondary to autoimmune hemolytic anemia due to hot antibodies. At the second admission, when reactivation occurred, the diagnosis was HPS secondary to a probable lymphoproliferative disease, unconfirmed by histology or autopsy.

Glucocorticoids (GC) were used in all patients and cyclosporine A (CSA) in 50\% of patients (table 2). In addition, supportive treatment such as transfusions of red blood cells and/or platelets were used almost in all patients. Broad-spectrum antibiotics were used in all patients except in 2 (Multiple Myeloma and HPS without defined etiology).

\section{Clinical characteristics and laboratory findings of all subgroups}

All patients presented high fever. Significant differences were observed in age, mortality, leukocytes, neutrophils, platelets, transaminases and total proteins. Age at diagnosis of HPS was lower in AI [40 (26.5 - 56.3)] and Infection subgroups [45.5 (30 - 61); p 0.001)]. The HM subgroup had severe cytopenias [platelets 4500 (650 - 15,750; p 0.009), leukocytes 2050 (20 - 728; p 0.0001) and neutrophils 0 ( 0 - 280; p 0.002)]. The Al subgroup had a highest elevation of AST [457 (289 - 1140; p 0.026). Tables 3 and 4 show the comparison of the 
demographic, clinical characteristics, laboratory findings, underlying disorders and mortality variables during hospital admission of the 5 subgroups.

\section{Clinical characteristics and laboratory findings of the Al and HM subgroups}

Compared the two most frequent subgroups, significant differences were observed according to the age, mortality, leukocytes, neutrophils, platelets, transaminases, total proteins, global hospital stay and hospital stay previous to the diagnosis by BM biopsy. The HM subgroup presented a higher number and more severe cytopenias (Figure 1, tables 3 and 4). The Al subgroup presented more severe liver abnormalities, such as higher elevation of AST [AI: 457 (289 1140) and HM: 106 (71 - 193); p 0.003] and ALT [AI: 432 (174 - 599) and HM: 109 (54 - 263); $p$ 0.017]. The HM subgroup had a higher global hospital stay [HM: 61.5 days (29.3 - 93.3) and Al: 30.5 days (9.5 - 53.3); p 0.036] and hospital stay previous to the diagnosis by BM biopsy [HM: 26 days (10 - 39) and Al: 10 days (5 - 16.5); p 0.038].

\section{Mortality:}

The overall mortality was $43.3 \%$ (Table 3 ). The HM subgroup had higher statistically significant mortality [8 patients (66.7\%)] compared to all subgroups ( $p$ 0.029 ) and compared to the Al subgroup [one patient (10\%); p 0.011)].

\section{Discussion:}

In our study the most frequent causes of secondary HPS were Al and HM diseases, similar to other published series. $(6,10,11,12)$ In our study we found that the most frequent causes of HPS were lymphomas, SLE and ASD, like in other Al or HM series published. $(13,14,15)$ The JIA was excluded from the study because the age. 
The third most frequent subgroup in our cohort was HPS without defined etiology (16.7\%). These patients were subjected to different diagnostic tests (X-rays, computerized axial tomography, analysis, serologies and bacterial cultures) during the hospital admission without concluding a final etiological diagnosis. The etiological absence of HPS is quite frequent in some series, being almost $30 \%$. (16) In our study, this subgroup had a greater, but not statistically significant hospital stays previous to diagnosis of HPS [(36 days (11.5-50; $p$ 0.191)] compared to all subgroups, and compared with Al and HM subgroups ( $p$ 0.067). In addition, this subgroup had a high mortality rate of $60 \%$ ( $p$ 0.029). The absence of an etiological diagnosis of HPS is likely to influence prognosis.

In our study we found only one case of HPS secondary to MST probably to the initiation of temozolomide. This event is caused by a loss of immune homeostasis induced by chemotherapy, aggravating T-cell dysfunction. $(13,16,17)$ The diagnosis of HPS secondary to chemotherapy is a challenged by the pre-existing neutropenia. In our opinion, HPS should be suspected in those patients who, after stopping chemotherapy, persist with neutropenia and fever that do not respond to broad-spectrum antibiotics.

The presence of infections as an etiology of HPS is less frequent compared to HPS secondary to HM and AI disease. Usually the infections are more related as a trigger, usually on an $\mathrm{Al}$ and $\mathrm{HM}$ disease. The most frequent viruses associated as a trigger are Epstein Barr (EBV), Cytomegalovirus (CMV) and Influenza. $(17,18,19)$ In our cohort we found 10 cases of infections related to HPS, 8 as triggers (2 CMV, 2 EBV, 2 respiratory infections probably not filiated and 2 bacterial infections) and 2 as primary etiologies of HPS.

In the $\mathrm{HM}$ diseases an adequate $\mathrm{T}$ and $\mathrm{NK}$ cell function is required for the elimination of antigenic and to end the inflammatory or autoimmune response. Aberrant activation of T and NK cells by lymphomas results in active, excessive, and sustained production of cytokines and macrophages, causing an HPS. The HPS also occurs in the course of other HM, such as B-cell lymphoma, Hodgkin's lymphoma, acute and chronic leukemias, acute myeloid leukemia, and myelodysplastic syndromes, as in our study. $(9,14,15,16)$ 
The clinical features reflect an alteration of the immune system, induced by a hypercitokinaemia caused by highly activated lymphocytes and macrophages. $(20,21)$ The clinical findings that suggest the presence of HPS are prolonged fever unresponsive to antibiotics and hepatosplenomegaly. The laboratory findings include the presence of cytopenias, which usually begin with a thrombocytopenia that progresses to severe pancytopenia. Other common findings include the presence of hyperferritinemia, hyponatremia, hypertransaminasemia, hypofibrinogenemia, hypertriglyceridemia, and hypoalbuminemia. (22) In our cohort, statistically significant differences were observed between the different subgroups in relation to the number and severity of cytopenias, being more severe in the HM subgroup. Also, a longer hospital stay previous to the diagnosis of HPS was observed in the HM subgroup compared to Al and Inf. subgroups, as observed in a Chinese study, which also showed an increased hypertriglyceridaemia and increased SCD25 activity in the HM subgroup. (23)

The triglycerides may not increase until the liver has been damaged for a long period of time; that is, hypertriglyceridemia is a sign of severe and late liver failure. In our study, no statistically significant differences were observed between the different subgroups (24). This may be due to the fact that the Al subgroup presented lower mortality and a greater liver alteration.

As we mentioned above, most of the patients included in our study presented an $\mathrm{Al}$ and HM disease, like in others published series $(3,6,10,12,13,16,17,25)$. We found a few studies in the literature that compare these two subgroup or differences that are not very relevant or significant. In our study we found in the comparative analysis between these two subgroups, that the HM presented a higher number and more severe cytopenias, in which plaquetopenia is the most importance. In addition, the HM subgroup presented a higher age at the time of diagnosis of HPS, a longer hospital stays, a longer hospital stays previous to the diagnosis of HPS and a higher mortality. The prognosis of the HM subgroup was worse, this may be due to the sum of several factors: such as the complexity of the disease itself, older age, a delay in the diagnosis of HPS and the severity of cytopenias, especially plaquetopenia. On the other hand, the Al subgroup 
presented a higher elevation of transaminases, probably due to a more systemic component, characteristic of Al diseases. In contrast, the HM subgroup had a more pronounced hypoproteinemia, probably related more to the severe liver damage.

There is a great heterogeneity in the prognosis of adult patients with HPS, the mortality rate varies between $20 \%$ and $90 \%$. $(11,13,25,26,27)$ In our study the overall mortality was $43.3 \%$, with significant differences according to the subgroup. In general, the malignant neoplasms underlying HPS, especially lymphoma, are considered an adverse prognostic factor. $(10,11,24,27)$.

The aim of this study is not to analysis the differences between MAS and a flare of an $\mathrm{Al}$ disease, but we thought to mention some relevant data given the great similarity between these two. So far, there is no clinical and laboratory pathognomonic finding that would help us to suspect in a MAS. (28-32) But, there are some clinical data that could help us to differentiate between these two, for example, the fever in MAS is continuous and in a flare is usually intermittent; hepatosplenomegaly can be present in both, but more often occurs in MAS; and, the alteration of the central nervous system and bleeding disorders are more frequents and severe in MAS. $(33,34)$ Also, the decreased of the platelet counts appear to be the most valuable data to differentiate, this finding being very suggestive of the appearance of a MAS. (35) A decrease in the levels of globular sedimentation rate (GSR) or a disproportion between GSR and C-reactive protein increases the suspicion of MAS. However, this finding occurs in advanced stages concomitant with hypofibrinogenemia. $(35,36,37)$

Generally, the current therapy is based on the combination of immunosuppressors and cytotoxics to treat the hyperinflammatory state, but the main goal of treatment is to control the underlying disease. In the HLH-94 and $\mathrm{HLH}-2004$ protocols the induction treatment is dexamethasone and etoposide, and for maintenance CSA. In refractory or recurrent cases, intrathecal methotrexate and allogeneic hematopoietic cell transplantation can be used. $(3,7,38)$ In the specific case of MAS the main treatment is GC, intravenous methylprednisolone at $30 \mathrm{mg} / \mathrm{kg} /$ dose (maximum $1 \mathrm{~g}$ ) for 1-3 days. If patients respond well, GC is reduced to $2-3 \mathrm{mg} / \mathrm{kg} /$ day in divided doses. In the absence 
of response to GC, it is recommended to add CSA $2-7 \mathrm{mg} / \mathrm{kg} /$ day. (39-41) In refractory cases to high dose GC and CSA, the HLH-2004 protocol should be considered. However, etoposide is not recommended in MAS due to its serious side effects, such as severe BM suppression, liver and kidney toxicity. (42) Biological therapies, such as IL-1 and IL-6 inhibitors, is recommended in refractory cases. (43.44) Other therapies such as intravenous immunoglobulin, cyclophosphamide, and plasmapheresis have provided inconsistent results. The treatments used in our patients do not differ from those reported in the literature. In the HM subgroup a greater number of treatments were used, probably due to the lack of early etiological diagnosis and the very complexity of the disease.

\section{Conclusions:}

We found that the HM subgroup presented a greater mortality, and more number and severe cytopenias, compared to all subgroups and especially with $\mathrm{Al}$ subgroup. The Al subgroup had a higher elevation of transaminases and a better prognosis than all subgroups. The HPS with no defined etiology subgroup presented a high mortality and a delay diagnosis of HPS.

\section{Bibliography:}

1. Risdall RJ, et al. Virus-associated hemophagocytic syndrome: a benign histiocytic proliferation distinct from malignant histiocytosis. Cancer 1979;44:993-1002.

2. Farquhar JW, et al. Familial haemophagocytic reticulosis. Arch Dis Child 1952;27:519-25.

3. Machaczka $M$, et al. Malignancy-associated hemophagocytic lymphohistiocytosis in adults: a retrospective population based analysis from a single center. Leuk Lymphoma. 2011;52:613-9.

4. Henter Jl, et al. HLH-2004: Diagnostic and therapeutic guidelines for hemophagocytic lymphohistiocytosis.Pediatr Blood Cancer. 2007;48:124. 
5. Kenneth L McClain, MD, PhD. Clinical features and diagnosis of hemophagocytic lymphohistiocytosis. UpToDate. July 29, 2015;

6. George MR. Hemophagocytic lymphohistiocytosis: review of etiologies and management. J Blood Med. June 2014; 5:69-86.

7. Kejian Zhang, MD, et al. Hemophagocytic Lymphohistiocytosis, Familial. GeneReviews. January 17, 2013.

8. Paul La Rose' e, et al. Recommendations for the management of hemophagocytic lymphohistiocytosis in adults. Blood. June 2019; 133: 23.

9. Laurence Fardet, et al. Development and validation of the HScore, a Score for the diagnosis of reactive hemophagocytic syndrome. Arthritis and Rheumatology. Vol. 6, No 9, September 2014, pp 2613-2620.

10. Riviere $S$, et al. Reactive hemophagocytic syndrome in adults: a retrospective analysis of 162 patients. Am J Med. 2014;127:1118-1125.

11. Parikh SA, et al. Prognostic factors and outcomes of adults with hemophagocytic lymphohistiocytosis. Mayo Clin Proc. 2014;89:484-492.

12.Schram AM, et al. Haemophagocytic lymphohistiocytosis in adults: a multicenter case series over 7 years. Br J Haematol. 2016;172:412-419.

13. Fukaya $S$, et al. Clinical features of haemophagocytic syndrome in patients with systemic autoimmune diseases: analysis of 30 cases. Rheumatology (Oxford) 2008;47:1686-91.

14. Lay JD, et al. Upregulation of tumor necrosis factor-alpha gene by EpsteinBarr virus and activation of macrophages in Epstein-Barr virus-infected $T$ cells in the pathogenesis of hemophagocytic syndrome. J Clin Invest. 1997;100:1969-79.

15. Tong $\mathrm{H}$, et al. Clinical characteristics of $\mathrm{T}$-cell lymphoma associated with hemophagocytic syndrome: comparison of T-cell lymphoma with and without hemophagocytic syndrome. Leuk Lymphoma. 2008;49:81-7.

16. Fei $\mathrm{Li}$, et al. Clinical characteristic and prognostic factors of adult hemophagocytic syndrome patients: a retrospective study of increasing awareness of a disease from a single-center in China. Orphanet Journal of rare Diseases (2015) 10:20

17. Otrock ZK, et al. Clinical characteristics, prognostic factors, and outcomes of adult patients with hemophagocytic lymphohistiocytosis. Am J Hematol. 2015;90:220-224. 
18. Ishii E, et al. Nationwide survey of hemophagocytic lymphohistiocytosis in Japan. Int J Hematol 2007;86:58-65.

19. Efthimiou P, et al. Life-threatening complications of adult-onset Still's disease. Clin Rheumatol 2014;33:305-14.

20.Behrens EM, et al. Occult macrophage activation syndrome in patients with systemic juvenile idiopathic arthritis. J Rheumatol 2007;34:1133-8.

21. Larroche C, et al. Pathogenesis of hemophagocytic syndrome (HPS). Autoimmun Rev. 2004;3:69-75.

22. Arico M, et al. Hemophagocytic lymphohistiocytosis. Report of 122 children from the International Registry: FHL Study Group of the Histiocyte Society. Leukemia. 1996;10:197-203.

23. Yinqun Guo, et al. Clinical features and prognostoc factors of adult secundary hemopahgocytic syndrome. Medicine. 2017. 96:22

24. Yanchun Zhao, et al. Risk factors of early death in adult patients with secondary hemophagocytic lymphohistiocytosis: a single-institution study of 171 Chinese patients. Hematology. 2019, vo. 24, No 1, 606-612.

25. Li J, et al. Hemophagocytic lymphohistiocytosis: clinical analysis of 103 adult patients. Medicine (Baltimore). 2014;93:100-105.

26. Li F, et al. Clinical characteristics and prognostic factors of adult hemophagocytic syndrome patients: a retrospective study of increasing awareness of a disease from a single-center in China. Orphanet $\mathrm{J}$ Rare Dis. 2015;10:20.

27.Zhou M, et al. Clinical features and outcomes in secondary adult hemophagocytic lymphohistiocytosis. QJM. 2018;111:23-31.

28. Batu ED, et al. Assessment of the HScore for reactive haemophagocytic syndrome in patients with rheumatic diseases. Scand J Rheumatol. 2017;46(1):44-48.

29. Yoon $\mathrm{JH}$, et al. Treatment outcomes and prognostic factors in adult patients with secondary hemophagocytic lymphohistiocytosis not associated with malignancy. Haematologica. 2019;104:269-276.

30. Bigenwald C, et al. A comprehensive analysis of Lymphoma- associated haemophagocytic syndrome in a large French multicentre cohort detects some clues to improve prognosis. Br J Haematol. 2018;183:68-75. 
31. Ravelli A, et al; Histiocyte Society. 2016 classification criteria for macrophage activation syndrome complicating systemic juvenile idiopathic arthritis: a European League Against Rheumatism/American College of Rheumatology/Paediatric Rheumatology International Trials Organisation collaborative initiative. Ann Rheum Dis. 2016;75(3):481-489.

32. Ravelli A, et al. Expert consensus on dynamics of laboratory tests for diagnosis of macrophage activation syndrome complicating systemic juvenile idiopathic arthritis. RMD Open. 2016;2(1): e000161.

33. Minoia F, et al. Development and initial validation of the Macrophage Activation Syndrome/Primary Hemophagocytic Lymphohistiocytosis Score, a diagnostic tool that differentiates primary hemophagocytic lymphohistiocytosis from macrophage activation syndrome. J Pediatr. 2017; 189:72-78.e3.

34. Kostik MM, et al. Identification of the best cutoff points and clinical signs specific for early recognition of macrophage activation syndrome in active systemic juvenile idiopathic arthritis. Semin Arthritis Rheum. 2015;44(4):417-422.

35. Assari R, et al. Dynamic changes, cut-off points, sensitive and specificity of laboratory data to differentiate macrophage activation syndrome form active disease. Dis Markers. 2015;2015:424381-424388

36. Emmenegger $U$, et al. Reactive macrophage activation syndrome: a simple screening strategy and its potential in early treatment initiation. Swiss Med Wkly. 2002;132(17-18):230-236.

37. Vilaiyuk $S$, et al. Recurrent macrophage activation syndrome as the primary manifestation in systemic lupus erythematosus and the benefit of serial ferritin measurements: a case-based review. Clin Rheumatol. 2013;32(6): 899-904.

38. Zuzana Tothova, et al. Hemophagocytic Syndrome and Critical Illnes: New insigths into Diagnosis and Management. J. Intensive Care Med. 2015 October; 30(7): 401-412.

39. Mouy R, et al. Efficacy of cyclosporine A in the treatment of macrophage activation syndrome in juvenile arthritis: report of five cases. J Pediatr. 1996;129(5):750-754. 
40. Ravelli A, et al. Macrophage activation syndrome in systemic juvenile rheumatoid arthritis successfully treated with cyclosporine. J Pediatr. 1996;128(2):275-278.

41. Quesnel B, et al. Successful treatment of juvenile rheumatoid arthritis associated haemophagocytic syndrome by cyclosporin $A$ with transient exacerbation by conventional-dose G-CSF. $\mathrm{Br} J$ Haematol. 1997;97(2):508-510.

42. Ravelli $A$, et al. Macrophage activation syndrome as part of systemic juvenile idiopathic arthritis: diagnosis, genetics, pathophysiology and treatment. Genes Immun. 2012;13(4):289-298.

43. Miettunen PM, et al. Successful treatment of severe paediatric rheumatic disease-associated macrophage activation syndrome with interleukin-1 inhibition following conventional immunosuppressive therapy: case series with 12 patients. Rheumatology. 2011;50(2):417-419.

44. Bruck N, et al. Rapid and sustained remission of systemic juvenile idiopathic arthritis-associated macrophage activation syndrome through treatment with anakinra and corticosteroids. J Clin Rheumatol. 2011;17(1):23-27. 
Figure 1: Demographic, clinical characteristics, laboratory findings and underlying disorders differences between $\mathrm{Al}$ (blue) and HM (orange) subgroups of Hemophagocytic Syndrome

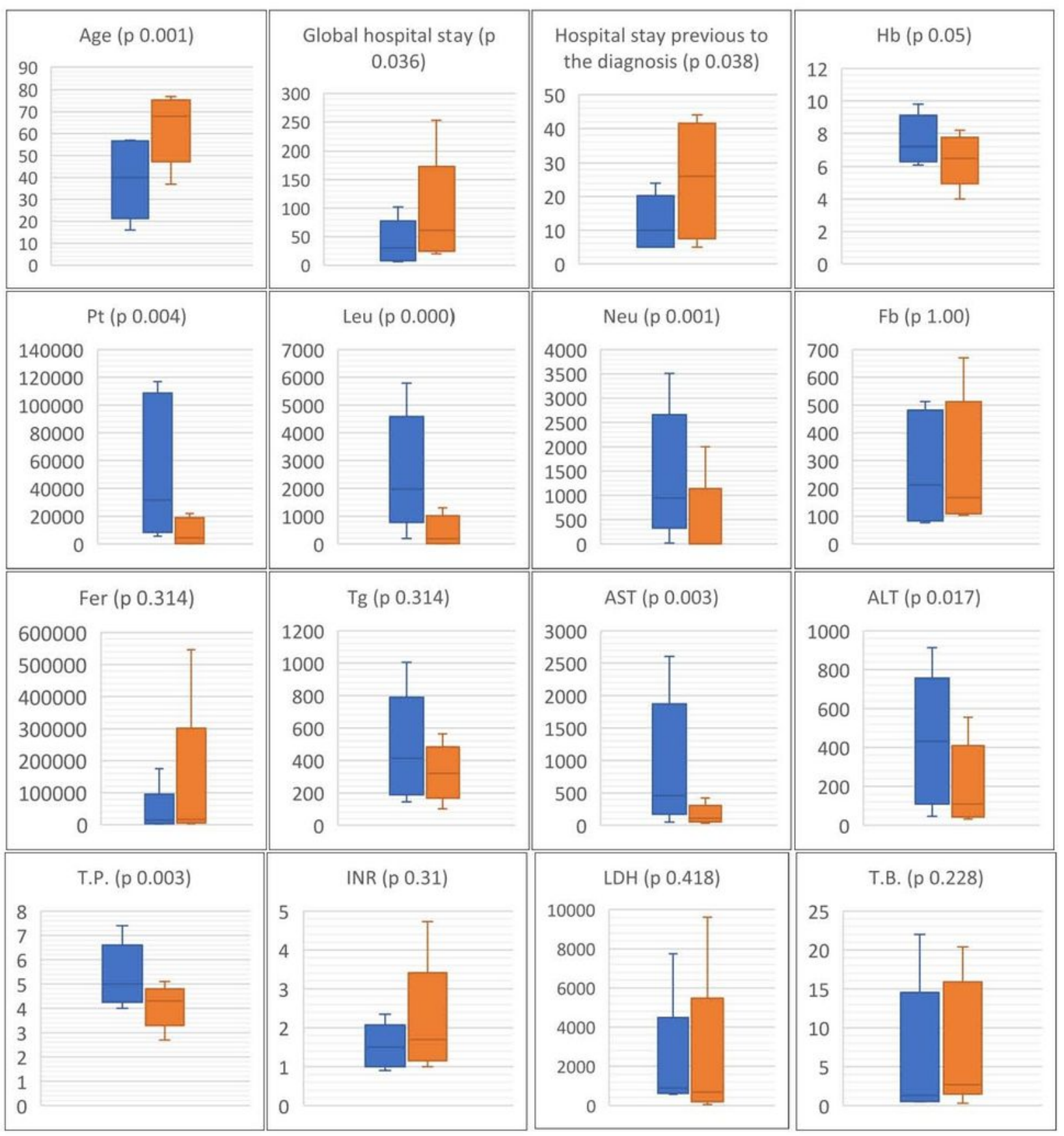

\section{Figure 1}

Demographic, clinical characteristics, laboratory findings and underlying disorders differences between Al (blue) and HM (orange) subgroups of Hemophagocytic Syndrome 